\title{
The Design and Implementation of Electromotion Curtain System using PLC and CDM Controller
}

\author{
Yeon-Gyu Choo, Sung-Soo Kang and Bong-Gi Kim, Member, KIMICS
}

\begin{abstract}
The electrical curtain is an electronic product which is most suitable for the sphere of intelligent home network. It provides the basic feature of intelligent home network such as the followings; various functions, powerful control. It also becomes the ideal technology with inexpensive power-line protocol, energy saving and solar power generation. Generally it is a suitable motor for BLDC to move the electrical curtain, because it provides the maintenance without difficulty, long-lived use with good efficiency. On this paper, we proposed the BLDC motor controller designed by CDM(Coefficient Diagram Method) and evaluated its performance with a simulation of the plant, designed the electrical curtain using BLDC motor and poweline protocol.
\end{abstract}

Index Terms-Electrical curtain, BLDC motor, Power-line protocol, $C D M$

\section{INTRODUCTION}

Recently, as the culture to pursue comfortable residential environment has spread, those apartment residential environment actively employing cuttingedge IT and information technology etc have been explosively increasing. In addition, it is judged that factors such as the pursuit of wellbeing culture and the reduction of energy costs will greatly affect future house designs and forms and life styles. Therefore, in order to introduce those house structures that maximize natural lighting and maximally utilize the energy of natural light, intelligent home related technologies that accommodate these functions are emerging as important technologies. Although electromotive curtains have been serving the function of a developed tool to just block sunlight so far, as well-being cultural demands have recently increased, they are in a tendency to be developed into composite home appliance systems combined with diverse sensors. In particular, with the rapid development of IT related technologies operated in linkage with home network systems, electromotive curtains are also required to be developed into intelligent curtain systems.

Manuscript received July 1, 2010 ; accepted July 1, 2010.

Yeon-Gyu Choo is with the Department of Electronic Engineering Jinju National University, Sung-Soo Kang and Bong-Gi Kim (Corresponding Author) are with the Department of Computer science \& Engineering, Jinju National University, Gyeongnam, Jinju, Korea (Email: bgkim@jinju.ac.kr)
Although intelligent home network systems provide many functions such as providing diverse conveniences and environments for comfortable residential culture, they a problem in that establishing the systems involves expensive costs.

Electromotive curtains can be implemented by applying communication methods using Power Modulation technologies instead of the existing TCP/IP based expensive communication methods since the control is not required to be fast,. Therefore, in order to implement the optimum electromotive curtain systems, the low cost power line communication related technologies in the Power modulation method that is currently emphasized in intelligent home network related areas and technologies to drive and control BLDC motors are necessary.

In this study, in order to control BLDC motors which are a core component of electromotive curtain systems, a controller in the Coefficient Diagram Method(CDM) will be designed and hardware will be implemented so that the motors can be controlled using power line network so that the performance can be identified.

\section{ELECTROMOTIVE CURTAIN SYSTEMS}

Electromotive curtains have a basic form of relaying on the power of a motor for the motions of curtains and they consist of motors for driving curtains and various kinds of curtain driving related devices.

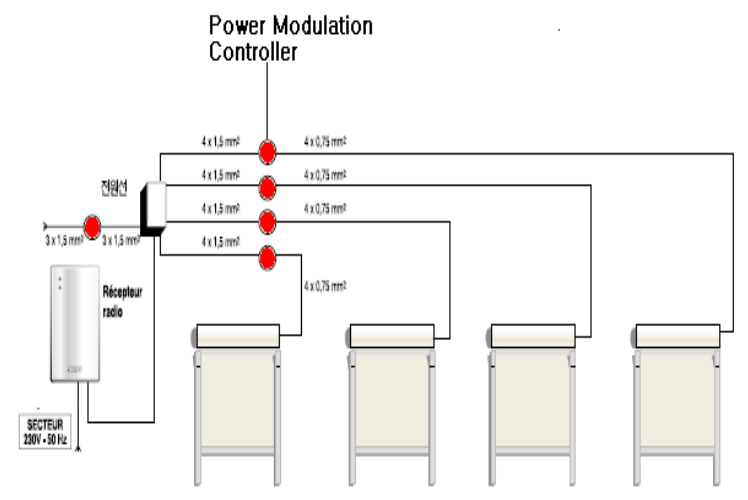

Fig. 1. Configuration diagram of the automatic electromotive curtain system 
In places where multiple electromotive curtains are operated such as buildings, offices and factories, a central control system is necessary to control the electromotive curtains. Mainly TCP/IP protocol or serial communication technologies are used to perform the central control function in general. Figure 1 shows the configuration diagram of the automatic electromotive curtain system to be implemented in this study.

\section{CONTROLLER DESIGN}

The equation of the BLDC motor that has one stator is as follows.[1,2]

$$
v_{1}=R_{1} i_{1}+p\left(L_{1} i_{1}+L_{21} i_{2}+\mathrm{L}_{31} \mathrm{i}_{3}\right)+e_{1}
$$

Where, $v_{1}$ is the voltage of the stator and $R_{1}$ is the resistance of the stator. Lis the inductance of the stator and $e_{1}$ is the back-EMF of the stator. The back-EMF can be expressed as a proportional equation of the angular velocity of the rotor.

$$
e_{1}=K_{E 1} \omega_{r(t)}
$$

Where, $K_{E 1}$ refers to the back-EMF constant. If the inductance of the three phase coil as the constant,

$$
\begin{gathered}
L_{1}=L_{2}=L_{3}=L_{s} \\
L_{21}=L_{31}=L_{32}=M
\end{gathered}
$$

Therefore, the equation of the three phase BLDC motor can be written as follows.

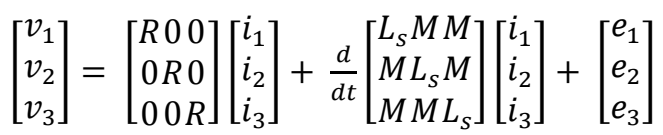

Where, $R$ is the resistance of the stator per phase and the values of the three phases are assumed to be the same.

If the current of the stator is maintained as $i_{1}+i_{2}+i_{3}=0$, equation (4) can be written as follows.

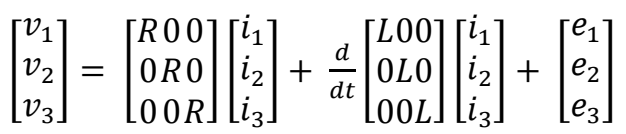

The power of the motor is as shown in equation (6).

$$
P_{\text {out }}=e_{1} i_{1}+e_{2} i_{2}+e_{3} i_{3}=T_{\mathrm{e}} \omega_{\mathrm{r}}
$$

The generated torque $T_{\mathrm{e}}$ can be written as per equation (7).

$$
\begin{aligned}
T_{e} & =e_{1} i_{1}+e_{2} i_{2}+e_{3} i_{3} \\
& =K_{\mathrm{e} 1} i_{1}+K_{\mathrm{e} 2} i_{2}+K_{\mathrm{e} 3} i_{3}
\end{aligned}
$$

Therefore, the equation of the BLDC motor can be defined as per equation (8).

$$
T_{e}=T_{L}+B \omega_{r}+J \frac{d}{d t} \omega_{r}
$$

Where, $T_{e}$ : generated torque, $T_{L}$ : load torque, $\omega_{r}$ : the angular velocity of the rotor, $J$ : the inertia of the rotor, $B$ : the coefficient of friction.

To identify changes in the dynamic characteristics of the BLDC motor applied to electromotive curtains and peripheral parameters through simulations, a controller designed based on CDM was applied first. The controller designed using CDM has stability and a tenacity against unit step responses. Figure 2 shows a basic configuration diagram of a controller designed using CDM for SISO(Single-Input Single-Output).[1,3]

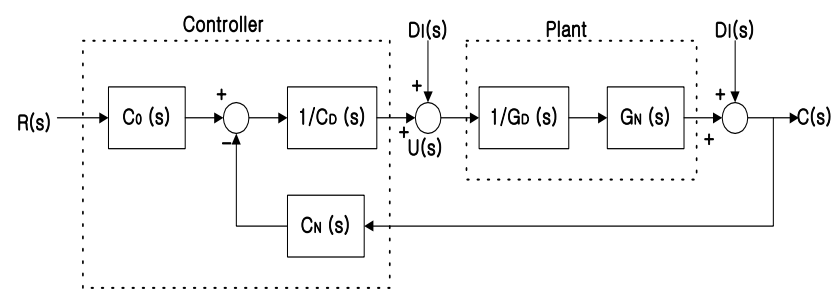

Fig. 2. Configuration diagram of controller using CDM

A polynomial expression of the characteristics of the control system shown in Figure 2 is as shown in equation (9).

$$
\begin{aligned}
P(s) & =C_{D}(s) G_{D}(s)+C_{N}(s) G_{N}(s) \\
& =a_{n} s^{n}+a_{n-1} s^{n-1}+\cdots+a_{0} \\
& =\sum_{i=0}^{n} a_{i} s^{i}
\end{aligned}
$$

The characteristics of the BLDC motor were simulated for a state where there was no load onto the CDM controller and another state where there was a load made by changing some parameters. Figure 3 shows the configuration diagram of the control system of the BLDC motor applied to the CDM controller.

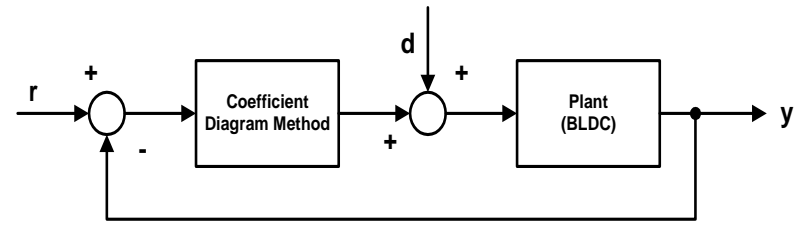

Fig. 3. Configuration diagram of BLDC Motor 
While the results of the simulation of the state of no load showed fast velocity responses and relatively small overshoots as shown in Figure 4(a), when the state was changed to have loads by changing the values of parameters $\mathbf{J}$ and $\mathrm{R}$, the results showed slightly slower velocity responses and large overshoots as shown in Figure 4(b). When designing an electromotive system, parts should be selected considering these characteristics of BLDC motors and appropriate parameters should be selected through CDM based controller simulations for appropriate velocity control.

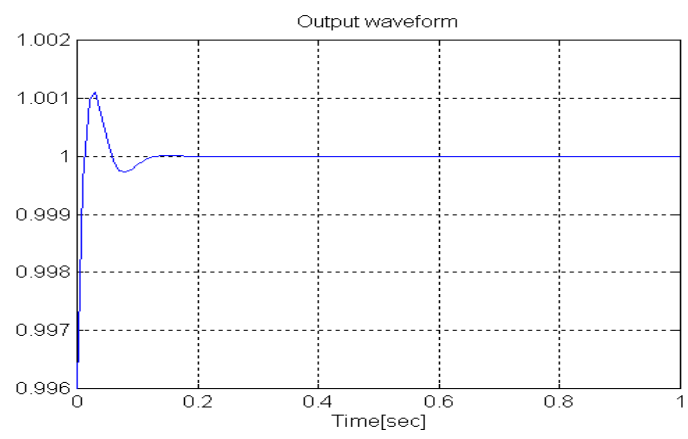

(a) Unload

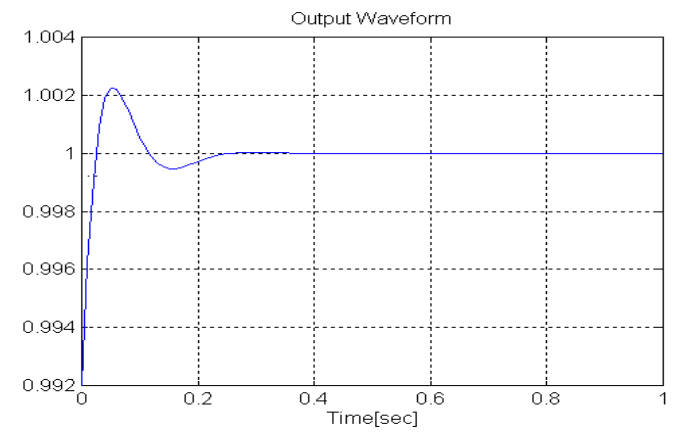

(b) Load (with parameter change)

Fig. 4. Velocity response of BLDC motor using CDM controller

\section{ELECTROMOTIVE CURTAIN SYSTEM DESIGN}

Basic components constituting an electromotive system can be subdivided into a motor to move curtains up and down, a motor driving and controlling part, a central control system that generally controls individual electromotive curtains and a communication part that implements smooth communications. Figure 5 shows a motion block diagram of the proposed electromotive curtain system.

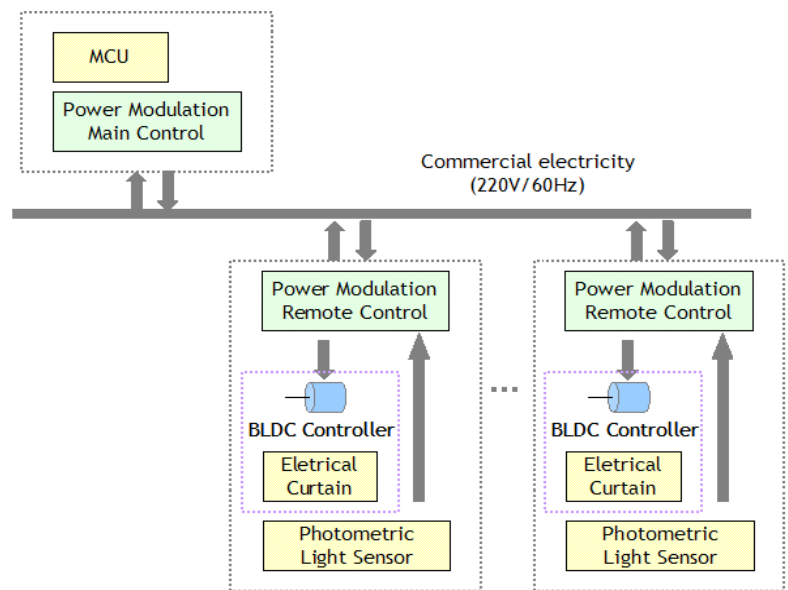

Fig. 5. Block diagram of proposed electromotive curtain system.

\section{4-1. Design of AC-DC buck converter}

In designing the AC-DC Buck Converter, before implementing the overall design processes, converter related simulations were performed in PSpice using a library model and the values of the error amplifier were applied by obtaining optimized parameters after evaluating the characteristics of the circuit. First, to design a power supply device in a switching mode, hardware was designed considering the major matters considered earlier. In particular, to minimize the size of the circuit mounted when a prototype would be made, related circuits were optimized using dedicated ICs etc. The prototype is operated by sending the alternating voltage inputted using a SG6841 dedicated IC from ICSGS-Thomson Co. through a built-in PWM controller to turn on-off the external switching element and using the alternating voltage again to output necessary direct voltages through a transformer. The power factor improvement circuit used to minimize the ripples caused by the inputted alternating voltage in the AC-DC conversion circuit was designed using an IC dedicated to Power Factor Correction. The L6561 sold by SGSThomson Co. is an IC designed to efficiently improve power factors in diverse devices using general alternating voltages. In particular, it has a built-in Low Pass Filter inside it that provides a characteristic of being strong against signals inputted from current sensors.

\section{4-2. BLDC drive and control circuit design}

Commonly used DC motors have excellent controllability and their controllers' economic efficiency is excellent and thus they are adopted by most electromotive curtains being imported.[4] However, they have shortcomings such the necessity of continuous maintenance in relation to the wear of brushes, the problem of heating by rectification and related cooling, difficulties in maintaining the condition for the stability of brushes and instability. On the other hand, the BLDC(Brushless DC) motor applied in this study does 
not require any brush as rectification is done by semiconductor control thus its maintenance is easy and it does not make mechanical noises. Therefore, it is frequently used for high speed rotations and also applied to diverse areas including conditions where there are many environmental restrictions in particular. $[5,6]$

The driving methods for BLDC motors are divided into a half wave electricity applying method that drives the motors by applying three phase sinusoidial waves and uses the periods existing only in the positive direction of alternating voltages and a whole wave electricity applying method that uses periods in both of positive and negative directions. The deriving methods can be also subdivided to several kinds based on exciting methods. In this study, the whole wave electricity applying method and the three phase exciting method as a driving method and the BLDC motor used is a product of Sewoo Industrial Systems Co., Ltd. Major specifications of the motor are as shown in Table 1 .

TABLE 1.

SPECIFICATION OF BLDC MOTOR

\begin{tabular}{|c|c|c|c|c|c|}
\hline Model & Voltage & Output & Current & Torque & Velocity \\
\hline $\begin{array}{c}\text { BD60- } \\
\text { N02405 } \\
0\end{array}$ & $24 \mathrm{~V}$ & $50 \mathrm{~W}$ & $3.5 \mathrm{~A}$ & $0.65 \mathrm{kgf} \cdot \mathrm{cm}$ & $5,000 \mathrm{rpm}$ \\
\hline
\end{tabular}

The BLDC motor drive and control circuit was designed by combining ICs dedicated to drive and micro controllers. This method can complement diverse problems in other methods and in particular, this method is advantageous in that speed controls can be easily implemented using the self PWM ports provided by micro controllers while this method can be applied to interfaces with various kinds of sensors.

Major components used in the BLDC drive and control circuit are the ATmega8 micro controllers of ATMEL Co. and the MC33035 of Motor Roller Co.,. The ATmega8 is a low power type high speed micro controller in the RISC structure that operates at speeds between $16 \mathrm{Mhz}$ and 16MIPS. It has $8 \mathrm{~K}$ bytes of program memories, a 512 byte EEPROM and a $1 \mathrm{~K}$ byte SRAM built inside of it and it is frequently applied to small embedded systems in industrial fields. In addition, since it provides an advantage of easy upgrades of firmware by providing an ISP(In System Programming) function, it can be easily upgraded. In addition, since it has three PWM output terminals, a four channel A/D converter with a resolution of 10 bits and a programmable USART etc, it can be said to be an ideal micro controller in designing and producing electromotive curtains. The MC33035 of Motor Roller Co., is an IC dedicated to BLDC drives designed to be applicable to three phase or four phase motor control systems. It uses drive voltages within the range of $10-30 \mathrm{~V}$ and it can drive external three phase MOSFET bridges using its internal high current drive circuit. If it cannot operate due to internal heat, it stops the operation by itself and either $60^{\circ}$ or $120^{\circ}$ can be selected as the topology of hole sensors to be used.

\section{4-3. PLC circuit design}

Designing home network systems using existing communication methods involved many problems in the aspect of economy and installations. In particular, in the case of dedicated lines and wireless communications, it was difficult to apply them due to diverse problems such as difficulties in installations and the acquisition of lines, costs for installations, maintenance and frequency allocations and thus networks are established when they are installed in most cases. Power Line Communication(PLC) is one of the communication methods recently that have been in the limelight recently as this method complements the aforementioned problems and makes the establishment of home network systems easy even in environments where no networks have been established.

In the case of power line communication circuits, the design of the line coupler is the most important part. The line coupler serves the role to separate information signals included in power source signals at $220 \mathrm{~V}, 60 \mathrm{~Hz}$ and its basic structure is as shown in Figure 6. The capacitor connected to the primary side of the transformer works with large impedance to power source signals and with small impedance to information signals (several hundred $\mathrm{kHz}$ ) thereby serving the role to insulate so that power source signals would not be absorbed by the modem. On the other hand, the L value of the primary side of the transformer has some impedance to information signals thereby transmitting the signals to the modem at the secondary side of the transformer. In this case, diverse circuits are added in relation to the filtering of signals going from the power source to the modem.

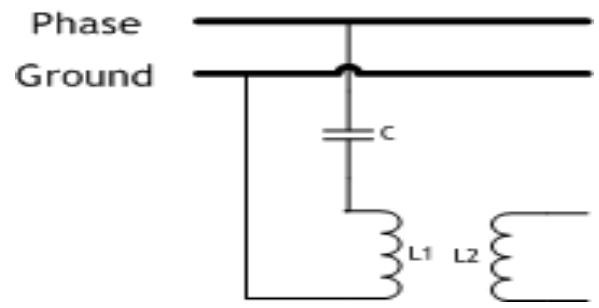

Fig. 6. Configuration diagram of line coupler

The most ideal filter should be designed to be in the form of a band passing filter when signals go from the power line to the modem and operate in the form of a band passing filter when signals are outputted from the modem to the power line on the contrary. However, it is not easy to implement a realistic ideal filter with hardware. Therefore, this problem will be minimized by designing a dedicated IC that maximally complements this problem. A Home Automation Modem called TDA5051 produced and sold by Phillips Co. was used to configure a power 
line modulation circuit and the circuit diagram is as shown in Figure 7.

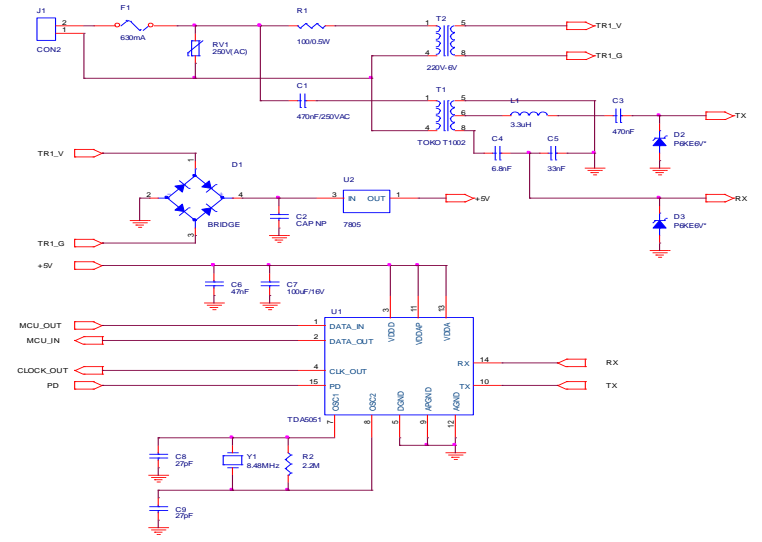

Fig. 7. Configure a power line modulation circuit

\section{4-4. Control circuit design using micro controllers}

A core component in electromotive curtains that serves the roles of driving BLDC motors, receiving and processing signals inputted by external circuits and sending the motion orders of electromotive curtains or monitoring the states of electromotive curtains through PLC modulation circuits is the micro controller. As explained earlier, because of its advantages of being able to not only drive BLDC but also implement diverse controls such as speed controls in BLDC drive circuits, its is essentially included in various kinds of circuits. Like the case that is applied to most embedded systems, first, the micro controller applied to electromotive curtains was selected and then, basic input/output operations and special functions were sufficiently tested using the development tools used in pre-development stages before designing the circuit of the system to be developed. In this study, two micro controllers were selected and used. The 80196 micro controller of Intel Co., was designed to be used as a master controller to control and monitor the produced individual prototypes of electromotive curtains from the center and the AVR micro controller of ATMEL Co., was designed to be mounted onto individual electromotive curtains to have diverse functions such as collecting data from various kinds of sensors, artificial intelligence type operations, checking of the states of the movements of the curtains, sending monitoring data, driving BLDC drive circuits and speed controls.

\section{ELECTROMOTIVE CURTAIN SYSTEM HARDWARE IMPLEMENTATION}

The AC-DC converted designed based on the Buck Converter provides currents sufficient to drive BLDC motors and the power for the operation of the BLDC drive circuit and the power line modulation circuits through the regulator. The power factor was tested using an IC dedicated to the improvement of power factors and the results showed the efficiency of at least $80 \%$. The drive board designed and produced in order to drive BLDC motors was made to fit to the performance and dimensions of the proposed BLDC and it was designed not only for motor operations but also for support as software by mounting the ATmega8 controller of ATMEL Co., inside of it. Complicated parts in terms of hardware such as the designation of forward/backward rotations of the motor and rotation speed control etc were simplified through the micro controllers. Figure 8 shows the BLDC drive board with a built-in micro controller ATmega8.

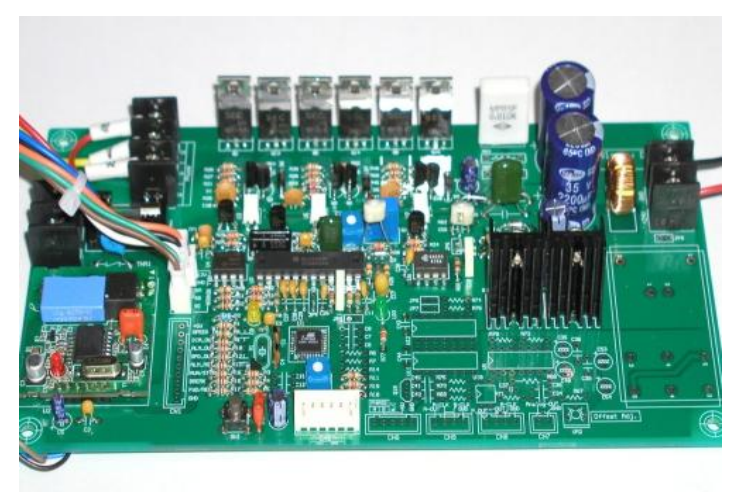

Fig. 8. BLDC drive board

The power line modem that provides the function of power line modulation was tested by mounting it on the right side of the BLDC drive as shown in Figure 8 in order to test electromotive curtains. The sending/receiving speeds of the power line modulation circuit is the sending speed provided by the ASK modulator TDA5051 which is $600 \mathrm{bps}$ and it was identified that data could be sent/received within one second by an experiment. The maximum number of accessible nodes was designed as 32 because of the CRC codes used to correct errors occurring in data formats and sending.

\section{CONCLUSION}

In this study, the characteristics of BLDC motors which is a major part used in electromotive curtain systems were theoretically approached and identified by performing simulations using a CDM mode controller in the state of no load and in the state of loading separately.

In addition, the proposed electromotive curtain system was designed as a system that enables remote and autonomous operations by linking with low cost power modulation and power line communication technologies differentiated from existing network technologies and the design was implemented with hardware. Through the acquisitions of the proposed power supply device, BLDC 
motor drive and power line modulation technology, the possibility to overcome restrictions in terms of installations and cost could be identified.

It is judged to be possible to produce power line network based electromotive curtain systems that are equipped with built-in power line modulation circuits to send information from various kinds of sensors such as sunlight/wind volumes or the state of the operation of curtains or receive operation orders from the central system to operate individually can be produced for all electromotive curtains. In particular, if an electromotive curtain system that can provide optimum environments is developed by introducing and applying artificial intelligence type algorithms to automatically operate based on sunlight, wind volumes, humidity and temperatures and applying sensibility ergonomics, it could be commercialized.

\section{REFERENCES}

[1] D. Isarakorn, T. Benjanarasuth, J. Ngamwiwit, N. Komine, "Speed Control of Ultrasonic Motor using PDF controller designed by CDM", pp.1378-1383

[2] Young-Sik Kim, Sung-Joong Kim, "Design of a Fuzzy P+ID controller for brushless DC motor speed control", ICCAS 2004, pp.627-630

[3] Photong Pathm Ngamwiwit Jongkol, Tirasesth Kitti, Komine Noriyuki, Manabe Shunji, "PIDA Controller Design by CDM", Proc. of 13th, KACC, 1998, pp. 395-400

[4] E. J. Davison, "A Method for Simplifying Linear Dynamic Systems", IEEE Trans. Automatic Control, vol. AC-11, pp.93-101, JAN 1966.

[5] K. Ogata, Modern Control Engineering, 2nd edition, Prentice Hall, 1990.

[6] C. C. Hang, K. J. Äström, and W. K. Ho, "Refinements of the Ziegler-Nichols tuning formula, Proc. IEE, Pt.D., vol. 138, pp. 111118,1991

[7] S. Ushakumari, "Adaptive Neuro-Fuzzy Controller for Improved Performance of a Permanent Magnet Brushless DC Motor", IEEE 2001, pp.493-496

[8] Wicharm Ch., Napatpong Suk., Taworn Benj. and Jongkol Nga, "Fuzzy I-PD controller for level control", SICE0ICASE International Joint Conference 2006, Busan, Korea, pp. 5649-5652.

[9] P. N. Paraskevopoulos, "On the design of PID output feedback controllers for linear multi-variable systems", IEEE Trans. on Industrial Electronics and Control Instrumentation, vol. IECI-27, pp. 16-18, Feb, 1980.

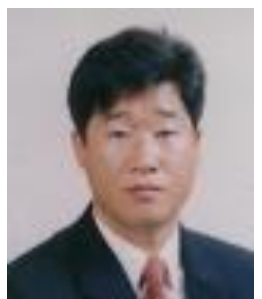

Yeon-Gyu Choo Received B.S. degree of Electronic Engineering in 1988 from Pukyong National University. and M.S. and Ph.D. degrees of Electronic Engineering in 1991 and 1997 respectively, from Dong-A University. In 1997, he joined the Jinju National University in Jinju, Korea, where is currently an professor and his research interests are in Intelligent Control System, Fuzzy Control System.

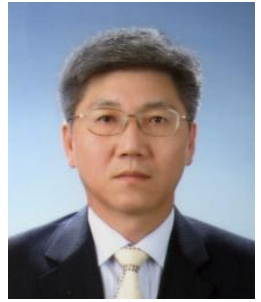

Sung-Soo Kang He received the B.S., degrees in computer science from Hongik University, Korea in 1981, and M.S. degrees in computer science from Busan National University, Korea in 1987 and $\mathrm{Ph} . \mathrm{D}$. degrees in computer science from Gyeongsang National University, Korea in 2007. Since 1988, he has been with Jinju National University, Korea, where he is currently professor in the Division of Computer Science \& Engineering. His research interests include Artifical Intellience, ERP.

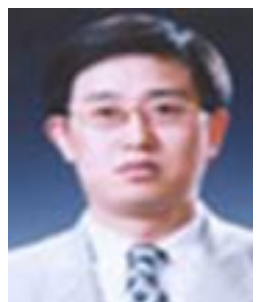

Bong-Gi Kim He received the B.S., M.S., and $\mathrm{Ph} . \mathrm{D}$. degrees in computer science from Soongsil University, Korea in 1987, 1989, and 1999 respectively. Since 1999, he has been with Jinju National University, Korea, where he is currently professor in the Division of Computer Science \& Engineering. His research interests include Multimedia System, Home Networking and Mobile System. 